\title{
Collection and dissemination of data from environmental monitoring systems in estuaries
}

\author{
Erwan Garel \\ Centre for Marine and Environmental Research (CIMA) University of Algarve, \\ 8005-139 Faro, Portugal \\ egarel@ualg.pt
}

\begin{abstract}
Environmental monitoring stations providing high frequency data over a multiyear time frame are not common in estuaries. These systems are designed to record extended timeseries at high frequency that are of great value for decision makers and the scientific community. However, the continuous acquisition of good quality data at estuaries is generally challenged by harsh environmental conditions. This contribution describes the main issues for continuous valid data (water quality and currents) acquisition in 2008-2014 with a monitoring station deployed at the Guadiana Estuary and how both near real-time and post-processed data were disseminated using web interfaces.
\end{abstract}

Keywords - water quality; hydrodynamics; estuary; monitoring programme; web interface.

\section{INTRODUCTION}

Estuaries are ecosystems with high productivity and tremendous ecological value. These systems are submitted to increasing environmental pressure from a wide range of sources, related mostly to anthropogenic activities (e.g., water flow regulation, land reclamation, watershed use, coastal development, and commercial and recreational activities). To maintain a balance between exploitation and conservation, the timely identification and remediation of any negative effects on estuary health produced by these activities requires the close monitoring of environmental parameters [1,2]. Extended periods of observations are generally needed in order to confidently distinguish between anthropogenic effects and natural trends (often highly variable) over the long-term [3]. Furthermore, high sampling intervals (hourly and less) are desirable to adequately resolve the complex bio-physical processes that control the health of estuaries and generally vary over short time scales (e.g., tide and episodic weather events) [4].

To address the above issues, autonomous systems capable of monitoring continuously environmental parameters at high sampling rates are increasingly deployed in estuaries, at least in highly-developed countries [5]. These systems can provide large and valuable datasets with wide potential applications for both decision makers and the scientific community. What makes these data interesting for scientists is the possibility to get continuous records at relatively high acquisition rates during long periods of time (weeks, months or years), which generally encompass very distinct external forcing. The data also support environmental monitoring programs for the establishment of reference conditions and the suitable identification of disturbances, for example in the case of accidental industrial pollution. However, despite the attractive features that monitoring systems installed at estuaries can provide, there are little reports that describe (1) the challenged faced with such deployments over a long time (e.g., months, years) and (2) possible schemes for broad dissemination of the recorded data.
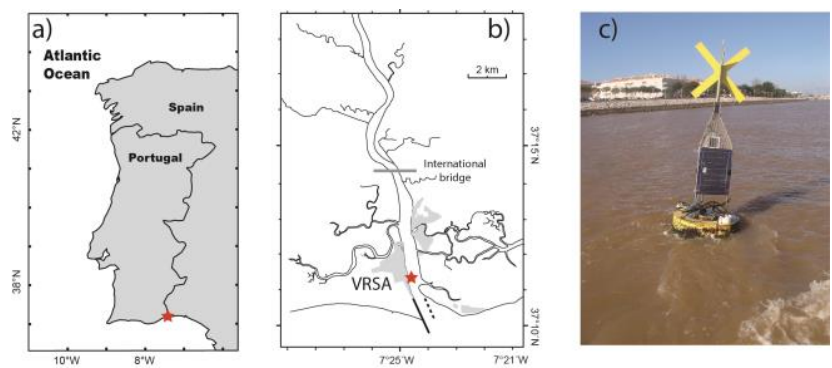

Fig. 1. General location (a) of the SIMPATICO System (red star) at Vila Real de Santo Antonio (VRSA) near the mouth of the Guadiana Estuary (b), and (c) photograph (E. Garel) of the surface buoy during a period of high river discharge in January 2010.

The SIMPATICO (integrated System for In-situ MultiPArametric moniToring In COastal areas) station has been operating since 2008 at the lower Guadiana Estuary (southern Spain - Portugal border) for the continuous in-situ monitoring of currents and water quality (Fig. 1). The primary motivation for the deployment of the system at this location was to perform a multi-parameter and multi-scale real-time environmental monitoring in a context of growing anthropogenic pressure. The latter is related mostly to strong flow regulation by the Alqueva dam, the largest dam in SouthWestern Europe, located at only $60 \mathrm{~km}$ upstream from the estuary head [6-8]. Similar monitoring stations were also deployed concurrently at the Tejo and Mondego estuaries but for other purposes and shorter duration [7]. Since 2014, the SIMPATICO operates intermittently, depending of specific research project requirements. In the present contribution, the main issues encountered with continuous data collection between 2008 and 2014 are described, together with the schemes used to disseminate near real-time and post-processed data to potential users. 


\section{SYSTEM OVERVIEW}

The SIMPATICO system consists of a surface floating buoy anchored to the seabed. The monitoring equipment includes a multi-parameter probe (YSI 6600 V2-4) for water quality fitted within the buoy and a bottom-mounted Acoustic Doppler current Profiler (750-kHz ADP, Sontek Argonaut XR) (Fig. 2). A brief overview of the system is provided below (for a detailed descriptions, see [7]).

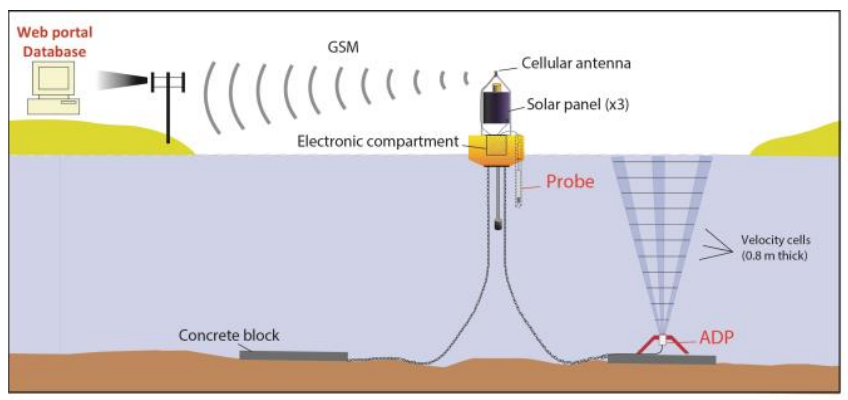

Fig. 2. The SIMPATICO system at the Guadiana Estuary.

The buoy includes a waterproof electronics compartment that houses a data logger (Campbell CR1000) and two 12 Volts batteries. The batteries are recharged by solar panels located on top of the buoy, enabling the system to operate in continuous sampling mode. Data records can be retrieved from the station using direct cable connection to a personal computer. In addition, the data logger is equipped with a modem enabling the transfer of data to a remote station through Global System for Mobile Communications (GSM).

The multi-parametric probe provides observations of chlorophyll concentration $\left(\mu \mathrm{g} \mathrm{l}^{-1}\right)$, both saturated $(\%)$ and dissolved $\left(\mathrm{mg} \mathrm{l}^{-1}\right)$ oxygen levels, turbidity (NTU), temperature $\left({ }^{\circ} \mathrm{C}\right), \mathrm{pH}$ and conductivity $\left(\mu \mathrm{S} \mathrm{cm}^{-1}\right)$ near the surface at hourly intervals (the highest sampling rate can be set to $4 \mathrm{~s}$ ). All sensors (except the temperature one) require regular calibration which was performed approximately every 3 months using buffer solutions in the range of variation of the measured parameters (see [9]).

Bottom-mounted at a mean water depth of $9 \mathrm{~m}$, the ADP measures the flow (velocity and direction) along the water column, pressure (water level) variations and near bed temperature. Additional parameters (listed in [9]) are available to diagnose the quality of each records. The size of the velocity cells was set to $0.8 \mathrm{~m}$ and the sampling interval to $15 \mathrm{~min}$.

\section{DATA ACCESS}

\section{A. Real time data}

Automatic and remote data access through telemetry is one of the main assets of the SIMPATICO system. The data were downloaded as tables (ASCII files) by a central server personal computer using a packbus network communication protocol, and exported to a database. These operations were performed automatically on a daily basis, but distinct time intervals, including real-time, can be set up. The parameters to download are user-defined. In addition to the probe and ADP records, the downloaded data also included the data logger status files, useful to check remotely the status of the system.

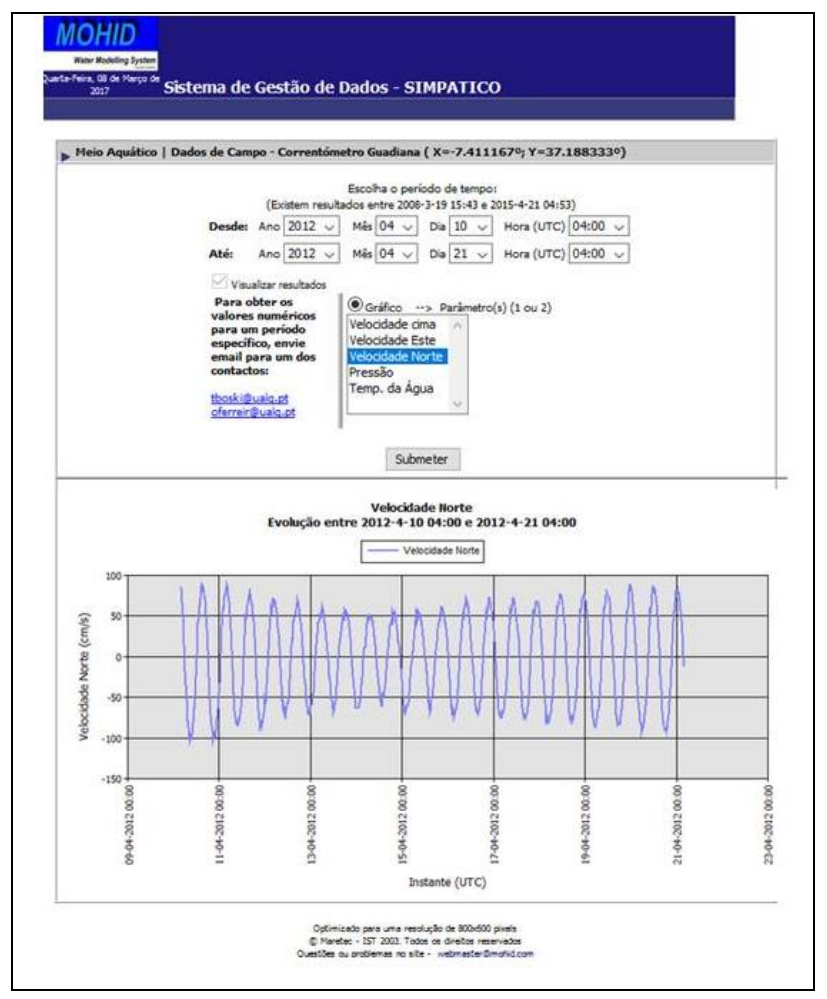

Fig. 3. Example of data access on the webportal of the SIMPATICO system (north component of the velocity, m/s, 10-21 April 2012).

The database is accessible through a web portal (http://webserver.mohid.com/simpatico/), where registered end-users can select parameters and time series for data visualization as graphs or tables (Fig. 3). No data screening and processing was performed at this stage. The main purpose of the web portal was to facilitate the quick visualization and download of the records for selected periods of interest.

Furthermore an automatic alarm system was developed to notify end-users by email when data were missing in the database or when measured parameters were out of (userdefined) selected ranges. This occurred in case of unusual environmental conditions, instrumentation flaws or system failure. Access to the (near real-time) data on the web portal generally permitted rapid identification of the cause for the alarms. In most cases, the alarm was triggered by erroneous measurements due for example to biofouling or sensor flaw (see [4]). The alarm system allowed the rapid maintenance of the station in the case of erroneous or missing records, hence reducing the temporal gaps of good (valid) data in the time series.

\section{B. Post-processed data}

The SIMPATICO system represents an important source of data for researchers investigating hydrodynamic and ecological processes acting in estuaries at time scales ranging from intratidal to seasonal. The recorded time-series also support the 
required development of comparative science based on similar observations at worldwide case studies.

For dissemination to a broad scientific audience, the entire dataset was deposited at the PANGAEA digital library (http://doi.pangaea.de/10.1594/PANGAEA.845750). The data are available publicly (under the Creative Commons Attribution 3.0 Unported License) in machine readable format (tab-delimitated text files), together with extensive information about the site, instrument, hardware, setup, and units. In addition, the data were comprehensively described in a support publication in Earth System Science Data, an international, interdisciplinary journal for the publication of articles on research data sets [9].

The published data consist of raw records with flags stating their quality status determined from thorough quality control procedures. All diagnostic parameters recorded by the ADP were also included, allowing a user to reprocess the data (as the requirements for data quality may depend on the intended use).

\section{DATA COLLECTION ISSUES}

Although more frequent in highly-developed countries, autonomous environmental monitoring stations are still not commonly deployed over a multiyear time frame in worldwide estuarine waters $[10,11]$. This is mainly because estuaries are harsh environments, where moorings may be submitted to strong currents, large waves' action, damage due to large size material carried along with the flow or fouling by life forms (generally at high growth rates). Additional threats to the instrumentation include mostly thefts and the risk of collision due to recreational or commercial boat activities within the area. Frequent visits to the site are generally required for system maintenance, which are often challenged by various factors such as underwater visibility, meteorological conditions, or boat and staff availability, amongst others. Gaps in the time series are unavoidable due to these imponderables difficulties to access to the system. The implementation and regular update of a detailed maintenance program is essential to prevent (as much as possible) interruption of the data records and to reduce their temporal extent.

Issues with the continuous records of ADP data from the SIMPATICO system were mainly related to strong physical conditions at the site. For example, the cable that links the ADP to the surface buoy broke twice in relation to strong flow conditions. In particular, a large flood in February 2010 severely damaged the whole system, producing a large gap in the time series until January 2012 (Fig. 4). ADP maintenance operations consisted mainly of scuba dives for the recovery or deployment of the damaged instrumentation. These operations were strongly dependent of visibility conditions (generally poor for several weeks following rain events). Overall, more than $99 \%$ of the ADP samples were validated as good, representing a cumulative time-series data of about 3.8 years (Fig. 4; see also the statistics in [9]).

Compared with the ADP, a much larger (i.e., frequent) maintenance effort was required in the case of the multiparametric probe to maintain the continuity of valid water quality data. The probe time series was interrupted by numerous temporal gaps, in particular in 2008-2010 (Fig. 4).
Several of these gaps were related to technical problems. However, the main concern for the continuous acquisition of good quality probe data was biofouling (e.g., Fig. 5a). Antifouling paint was applied around the sensors during the first year of the programme (2008), but did not increase significantly the length of valid measurements. As an example, biofouling over the chlorophyll optical lens often resulted in saturated records (i.e., up to maximum value) only one week after sensor cleaning. From February 2009 onward, an antifouling kit (consisting of copper-based mesh screens and protection tape) was used to protect more efficiently the sensors, increasing substantially their length of valid operation (but without resolving definitely the question). Using this kit, maintenance operations to clean the probe sensors were performed every 3 weeks, approximately, in order to obtain continuous valid records. Finally, during the entire monitoring period (from 2008 to 2014), the probe has been operating for about 3 years (i.e., 25,649 hourly records); during this time, each sensor has provided more than $65 \%$ of valid data, representing a cumulative time of about 2 years (Fig. 4). A detailed analysis and statistics of the validated data time span is provided in [9].

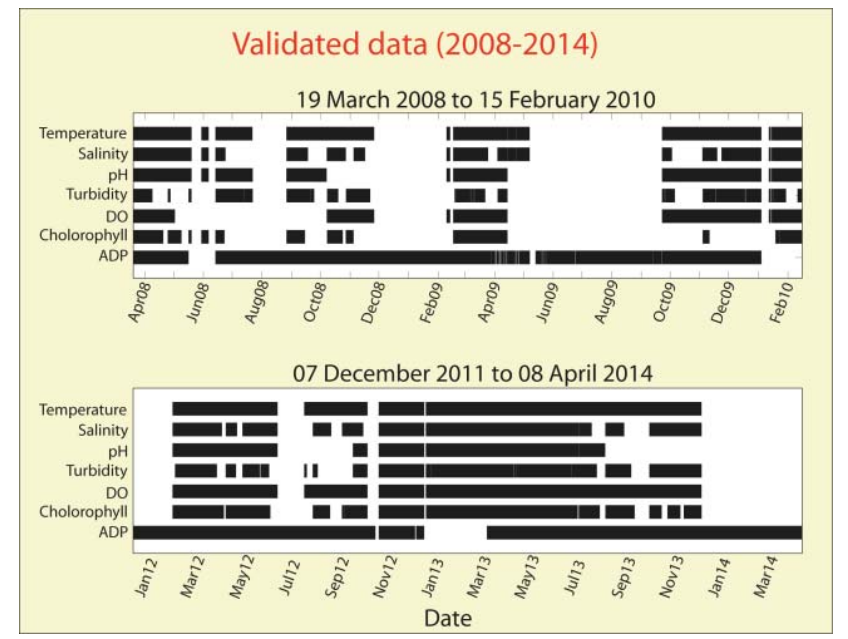

Fig. 4. Validated data (black). The time series consists of 2 main periods separated by a large temporal gap due a flood-induced system failure in February 2010.

\section{CONCLUSION}

The SIMPATICO system, an autonomous station for continuous water quality and current monitoring at high temporal resolution was operating from 2008 to 2014 at the Guadiana Estuary. Experience with this system shows that the continuous acquisition of good quality data over extended time periods (months, at least) requires substantial and regular maintenance efforts, in particular with regards to the multiparametric probe data. Nevertheless, relatively long continuous time-series with valid coupled water quality and hydrodynamic measurements were achieved, in particular in 2013-2014 with the help of the experience acquired during the preceding years. These remarkable datasets have potential applications for a wide range of end-users including scientists investigating ecohydrodynamic processes and management agencies. The collected data are particularly suitable regarding the 
implementation of monitoring programs and alarm systems, together with the development of comparative eco-hydrological science in an international context. With such broad and diverse potential end-user applications, data dissemination should be considered together with data quality as essential components of the successful implementation of autonomous monitoring systems in estuaries. As an example, the parameters monitored by the SIMPATICO station were provided as raw data in nearly-real time and as flagged (raw and quality checked) data using web interfaces and databases.
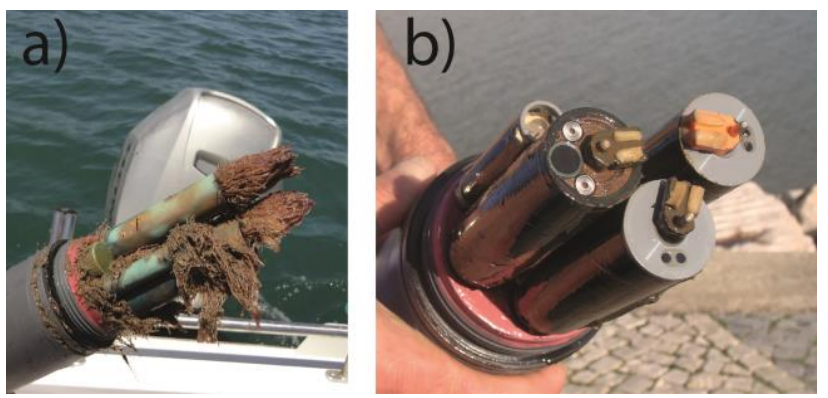

Fig. 5. Photographs (E. Garel) of the probe sensors illustrating various biofouling growth: (a) on the 12 July 2012, 5 weeks after the previous maintenance; (b) on the 20 December 2012, 1 week after maintenance.

\section{ACKNOWLEDGMENT}

The SIMPATICO monitoring station was purchased through a national program of the Portuguese Science and Technology Foundation (FCT) for the re-equipping of scientific institutions (Reeq/484/MAR/2005). The SIMPATICO web portal was developed and maintained by IST (Instituto Superior Tecnico) in Lisbon. The author acknowledges the help of all the staff who participated in the field maintenance of the SIMPATICO station.

\section{REFERENCES}

[1] M. J. Kennish, "Environmental threats and environmental future of estuaries," Environmental Conservation, vol. 29, pp. 78-107, 2002.

[2] M. Zalewski, "Ecohydrology: process-oriented thinking towards sustainable river basins," Ecohydrology \& Hydrobiology, vol. 13, pp. 97-103, 2013.

[3] T. Dworak, C. Gonzalez, C. Laaser, and E. Interwies, "The need for new monitoring tools to implement the WFD," Environmental Science \& Policy, vol. 8, pp. 301-306, 2005.

[4] E. Garel and Ó. Ferreira, "Monitoring estuaries using non-permanent stations: practical aspects and data examples," Ocean Dynamics, vol. 61, pp. 891-902, 2011.

[5] H. B. Glasgow, J. M. Burkholder, R. E. Reed, A. J. Lewitus, and J. E. Kleinman, "Real-time remote monitoring of water quality: a review of current applications, and advancements in sensor, telemetry, and computer technologies," Journal of experimental marine biology and ecology, vol. 300, pp. 409-448, 2004.

[6] J. M. A. Dias, R. Gonzalez, and O. Ferreira, "Natural versus anthropic causes in variations of sand export from river basins: an example from the Guadiana river mouth (southwestern Iberia)," in Rapid transgression into semi-enclosed basins, Gdansk, pp. 95-102, 2004.

[7] E. Garel, S. Nunes, J. M. Neto, R. Fernandes., R. Neves, J. C. Marques, et al., "The autonomous Simpatico system for real-time continuous water-quality and current velocity monitoring: examples of application in three Portuguese estuaries," Geo-Marine Letters, vol. 29, pp. 331-341, 2009.

[8] M. H. Guimarães, A. Mascarenhas, C. Sousa, T. Boski, and T. Dentinho, "The impact of water quality changes on the socio-economic system of the Guadiana Estuary: an assessment of management options," Ecology and Society, vol. 17, p. 38, 2012.

[9] E. Garel and Ó. Ferreira, "Multi-year high-frequency physical and environmental observations at the Guadiana Estuary," Earth Syst. Sci. Data, vol. 7, pp. 299-309, 2015.

[10] T. Chapin, J. Caffrey, H. Jannasch, L. Coletti, J. Haskins, and K. Johnson, "Nitrate Sources and Sinks in Elkhorn Slough, California: Results from Long-term Continuous in situ Nitrate Analyzers," Estuaries, vol. 27, pp. 882-894, 2004.

[11] M. Varney, Chemical Sensors in Oceanography vol. 1. Amsterdam: S. Gordon and Breach Science Publishers, 2000. 